fifth order of this reflexion. The fourth and fifth orders come to lie within the region of the $A_{1}$ interference of 'silk II', which is smeared out and is the inmost interference hitherto observed. In fact, $A_{1}$ has the same position in the lattice (that is, coinciding with the backbone-H-bond-grid) and seems to split, at least partially, into the new sharper interferences.

It seems possible to interpret the appearance of this large period within the direction of the sidechain on the grounds of absorption measurements with polarized ultra-violet light ${ }^{4}$. These experiments show that once 'silk III' has formed, the planes of the tyrosine residues are oriented in one plane containing the fibre axis. Closely connected with this orientation, an increase of phenolic dissociation of the tyrosine molecules occurs probably in consequence of formation of salt bridges or hydrogen-bonds.

According to Bergmann and Niemann ${ }^{5}$, every sixteenth amino-acid along the peptide-chain is a tyrosine residue. Furthermore, the large period in the direction of the aide-chain contains 8-10 peptidgrids, if one assumes the same steric arrangement of the peptide-chains for 'silk II' and 'silk III'.

There exist various possibilities of a steric correlation between these two facts, whereas the formation of a large period could scarcely be explained on the basis of the simple glycyl-alanine lattice assumed hitherto by many authors.

We have learned from a private communication that $H$. Zahn has observed similar changes in X-ray diagrams of silk treated with nitric acid.

\section{O. KRATKY}

E. SchaUtanstein

A. SeKora

Institute for Theoretical and

Physical Chemistry,

University, Graz.

' Nature, 165, 319 (1950).

'Kratky, O., Z. phys. Chem., (B), 5, 297 (1929). Kratky, O., and Kuriyama, S., Z. phys. Chem.; (B), 11, 363 (1931).

${ }^{3}$ Cf. Astbury, W. T., J. Chem. Soc., 337 (1942). Nowotny, H., and Chem., (B), 53, 61 (1943).

${ }^{4}$ Schauenstein, E., Fixl, J. O., and Kratky, O., Mh. Chem., 80, 143 (1949). Schauenstein, E., Mh. Chem., 80, 820 (1949).

${ }^{5}$ Bergmann, M., and Niemann, C., J. Biol. Chem., 122, 577 (1938).

\section{Viridin}

VIRIDIN, an antibiotic produced by a pigmentforming strain of the common soil fungus Trichoderma viride, was first described by Brian and McGowan ${ }^{1}$, and Brian, Curtis, Hemming and McGowan'. Viridin is not anti-bacterial but is highly antifungal. Its activity against certain fungi is remarkably high. Germination of the spores of, for example, Botrytis allii is prevented by a concentration of 0.019 p.p.m. of $\alpha$-viridin or $0 \cdot 156$ p.p.m. of $\beta$-viridin. Among synthetic fungicides, only the organo-mercurials are of the same order of activity.

Several methods have been tried for the isolation and purification of viridin. The procedure described here provides viridin in good yields and gives reproducible results. Viridin was extracted from the culture filtrate with chloroform. After evaporation of the chloroform extract under reduced pressure and treatment of the oily residue with ice-cold methanol, a crystalline, slightly coloured material was isolated.

Viridin reacts with ketonic reagents, although all attempts to obtain crystalline derivatives have so far been unsuccessful. However, viridin reacts readily with Girard's reagent ${ }^{3}$, by means of which separation of ketonic from non-ketonic material can be achieved. The ketonic part was further purified by chromatography (mothod of fractional elution). On neutral or weakly acid alumina, far-reaching degradation was observed. It was thus necessary to use a strongly acidic alumina column. On such a column, it was possible to separate viridin into $\alpha$ - and $\beta$-viridin, although the quantity of the latter compound was variable and usually of a very small order.

The $\alpha$-isomer was isolated from the benzene - ether eluate. After recrystallization from methanol, acetone and finally dilute acetic acid, $\alpha$-viridin was obtained as fine, colourless needles, decomposition point $208-217^{\circ} \mathrm{C}$, , determined on a Kofler block. $\alpha$-Viridin, which contains no nitrogen, no halogen, no sulphur and no phosphorus, does not decolorize bromine in the cold, produces no coloration with tetranitro-methane, gives no reaction with Schiff's reagent or 1:4-dihydroxy-naphthalene, but reduces Fehling's solution and Tollens's reagent in the cold. It does not give a positive iodoform reaction. With concentrated sulphuric acid, it produces an orangered colour, which on warming changes to a deep red. It is soluble in cold caustic soda, forming a red solution.

Analyses and molecular weight determinations reveal that the hitherto accepted molecular formula should be replaced by $\mathrm{C}_{19} \mathrm{H}_{10} \mathrm{O}_{8}$. Viridin contains one methoxy- but no acetyl-group as previously assumed. Zerewitinoff determinations disclose the presence of two active hydrogen atoms. On hydrogenation in glacial acetic acid in presence of Adam's catalyst or in alcohol with palladium charcoal, $7 \mathrm{~mol}$. hydrogen are taken up. $\alpha$-Viridin is optically active, $[x] D^{20}=-213 \cdot 4^{\circ}$ (chloroform); it shows two absorption bands in the ultra-violet: I, $>\max$. $3045 \mathrm{~A}$., $\varepsilon=13,200$; II, 7 max. $2413 \mathrm{~A}$., $\varepsilon=31,000$ (alcohol).

Further evidence for the empirical formula $\mathrm{C}_{10} \mathrm{H}_{18} \mathrm{O}_{6}$ was adduced by the preparation of a diacetyl derivative (m.p. $145-50^{\circ} \mathrm{C}$.), the analyses of which were in agreement with $\mathrm{C}_{23} \mathrm{H}_{20} \mathrm{O}_{8}$. From this a 2 : 4-dinitrophenylhydrazone (m.p. 271-73 ${ }^{\circ}$ was obtained which agreed with $\mathrm{C}_{29} \mathrm{H}_{24} \mathrm{O}_{11} \mathrm{~N}_{4}$, thus further confirming the revised formula for viridin.

$\beta$-Viridin was isolated from the chloroform eluates. After recrystallization from methanol $\beta$-viridin forms fine needles. $\beta$-Viridin has the same composition as $\alpha$-viridin and differs only in its optical rotation, $[\alpha] D^{20}=-50 \cdot 7^{\circ}$ (chloroform) and melting point $\left(140^{\circ}\right.$ C.). The two compounds also have identical absorption spectra: I, $\gamma_{\max } 3040$ A., $\varepsilon=13,200$; II, $\lambda_{\max .} 2415 \mathrm{~A}$., $\varepsilon=31,000$ (alcohol).

An attempt to separate the $\alpha$ - and $\beta$-isomer by paper chromatography was unsuccessful.

Details of this and further work will be published elsewhere. We are grateful to Dr. P. W. Brian and Mr. P. J. Curtis for their co-operation in supplying the starting material and in undertaking the bio. logical tests.

\section{E. B. VISCHER \\ S. R. HowLAND \\ H. RaUdNITZ}

Imperial Chemical Industries, Ltd.,

Butterwick Research Laboratories, The Frythe, Welwyn, Herts. Nov. 30.

1 Brian, P. W. and McGowan, J. C., Nature, 156, 144 (1945).

${ }^{2}$ Brisn P. W., Curtis, P. J., Hemming, H. G., and McGowan, J. C., risn P. W., Curtis, P. J, Hemming,

- Girard, A., and Sandulesco, G., Helv. Chem. Acta, 19, 1905 (1936). 